\title{
THE GEOMETRIZATION OF BIOLOGY: A CORRECTION
}

\author{
N. RASHEVSKY \\ CoMmitTeE on MATHEMATICAL Biology \\ The UNIVERSITy OF CHICAgo
}

An error in a previous paper (Bull. Math. Biophysics, 18, 31-56, 1956), pointed out by Professor R. L. Wilder, is corrected.

In a previous paper entitled "The Geometrization of Biology" (Rashevsky, 1956), we discussed some special types of topological spaces which have the following characteristic: The space $M$ is a subspace of another space $S$. The loss of a point $\beta$ of $M$ makes some points $\alpha_{1}, \alpha_{2}, \ldots, \alpha_{i}$ of $S-M$ acquire with respect to $M$ a property $P$ which those points do not possess unless the point $\beta$ is removed from $M$. The particular property $P$ considered in $l o c$. cit. is to make $M$ connected.

We have given in loc. cit. several examples of such spaces. Professor R. L. Wilder has pointed out to the author that one of the examples contains an error and is altogether wrong. Professor Wilder also showed the way to correct this error and to make the example valid, besides pointing out several minor errors.

On page 44 of loc. cit. we considered a case of a space $M$, which we called a $\lambda^{n}$-space, and which is described as follows:

Consider a family of circles in the plane $E^{2}$ :

$$
(x-\alpha)^{2}+y^{2}=\alpha^{2},
$$

where $\alpha=\frac{1}{2}$ or $\alpha$ is an irrational number between 0 and $\frac{1}{2}$. The cardinal number of such a set of circles is $\aleph$. Each circle intersects the line $y=0$ at $x=0$ and at $x=2 \alpha$. Remove in each circle the point $x=2 \alpha$. The space $M$ obtained by this removal of points from the family of circles (1), considered as a subspace of $S=E^{2}$, is connected. Removal of the point $O$, which we shall designate by $\beta$, from $M$ makes it not connected. 
Following the above description we stated on page 44 of loc. cit. that "in order to make it connected with the point $O$ removed, we must add to it all the points $x=2 \alpha$." This is obviously wrong, for even with all points $x=2 \alpha$ present, the removal of the point $O$ makes the family of circles homeomorph to a family of parallel straight lines, thus leaving it non-connected.

To make the example valid and to have the removal of the point $O$ make a non-countable number of points acquire property $P$, we proceed as follows:

In the family of circles (1), let $\alpha=\frac{1}{2}$ or $\alpha$ be equal to a rational number. The removal of the point $\beta$ makes the family of circles (1) non-connected. But now the addition of all points of the segment $(0,1)$ of the $x$-axis which do not lie on any circle of the family, that is, of all points with $x \neq 2 \alpha, 0<x<1, y=0$; will make the resulting space connected. The number of points to be thus added is non-countable, being the number of all irrational points between 0 and 1 . Thus the removal of the point $\beta$ makes all points $x \neq 2 \alpha, 0<x<1, y=0$ acquire the property $P$.

The $\lambda \cdots$ space described in paragraphs 4 and 5 on page 44 also does not satisfy the necessary conditions. Let $z=z_{1}$ be an irrational number, $0<z_{1}<1$. The portion of the space $S$ defined, for which $z>z_{1}$, call $A$; the portion for which $z<z_{1}$, call $B$; and the portion for which $z=z_{1}$, with the point $\beta$ of this set removed, call $C$. Then $A$ is connected, being topologically similar to $S$. But the irrational number $\beta$ is a limit point of the irrationals $\beta^{\circ}$. between $\beta$ and 1 , and correspondingly every point of $C$ is a limit point of $A$. Thus $A \cup C$ is connected, as it consists of a connected set $A$ plus limit points of $A$ (Wilder, 1949, p. 16, Theorem 7.2). Similarly $B \cup C$ is connected. Finally, $A \cup B \cup C$ is connected, being the union of two connected sets with points in common. Thus $S-\beta$ is not disconnected (lbid, Theorem 7.3).

If the example is modified so that each $\lambda^{\prime}$-space (in the amended sense) is in a plane which passes through the $x$-axis, in a sequence of planes with each two adjacent planes forming an angle $\pi / n$, where $n$ may be as large as we wish, then the point $\beta=(0,0,0)$ will be a disconnecting point, and the set of irrational points of the $x$-axis $(0<x<1)$ will acquire property $P$. This space we may call $\lambda^{\prime \prime}$-space.

The theorem stated on page 44 remains in force for those $\lambda^{\prime}$ and $\lambda$ spaces. However, for precision the theorem should read: "The 
analytical intersection of two $\lambda\left(\lambda^{\prime}, \lambda^{\prime \prime}\right)$ spaces is not a $\lambda\left(\lambda^{\prime}, \lambda^{\prime \prime}\right)$ space and is always not connected."

The following minor errors should be corrected:

Line 20, p. 42: The number of sets that can acquire property $P$ is not countably infinite; it is the same as the number of ways of selecting subsets of the $\beta_{i}$ 's...i.e., $2^{\kappa_{0}}=\aleph$.

Line 5, p. 43: Analogous remark.

Line 2, p. 44: The "set of sets" is of cardinal $\aleph$.

On this occasion we would like also to suggest a change in a sentence on page 47 of loc. cit., which, while not wrong, is ambiguous. The sentence on lines 7 to 10 from the top should read: "The removal of point $\beta_{1}$ from $M$ makes either the point $\alpha_{1}$ or the point $\alpha_{2}$ of $S-M$ acquire the property $P$; while the removal of the point $\beta_{2}$ makes either the point $\alpha_{1}^{1}$ or the point $\alpha_{2}^{2}$ of $S-M$ acquire the property $P$. If, however, after removal of $\beta_{1}, \alpha_{1}$ is added, then $\alpha_{2}$ loses the property $P$ and vice versa. The same hold about $\beta_{2}, \alpha_{2}^{1}$, and $\alpha_{2}^{2}$."

The author wishes to express his gratitude to Professor R. L. Wilder.

\section{LITERATURE}

Rashevsky, N. 1956. "The Geometrization of Biology." Bull. Math. Biophysics, 18, 31-56.

Wilder, R. L. 1949. Topology of Manifolds. New York: American Mathematical Society. 